\title{
Early maternal separation and the trajectory of borderline personality disorder symptoms
}

\author{
THOMAS N. CRAWFORD, PATRICIA R. COHEN, HENIAN CHEN, \\ DEIDRE M. ANGLIN, AND MIRIAM EHRENSAFT \\ New York State Psychiatric Institute
}

\begin{abstract}
Extended maternal separations before age 5 were evaluated as a predictor of long-term risk for offspring borderline personality disorder (BPD) symptoms in longitudinal data from a large random community sample. Early separations from mother predicted elevations in BPD symptoms assessed repeatedly from early adolescence to middle adulthood. Early separations also predicted a slower than normal rate of decline in symptoms with age. Other theoretically grounded risks were examined and shown to predict elevated BPD symptoms over the developmental trajectory. Long-term effects of early separations were largely independent of childhood temperament, child abuse, maternal problems, and parenting risks. These data provide the first prospectively collected data on the developmental course of BPD symptoms and suggest a series of environmental and other influences on these very disabling problems.
\end{abstract}

Borderline personality disorder (BPD) is a serious psychiatric disturbance characterized by emotional lability, poor impulse control, and angry outbursts. In addition to core disturbances in affect regulation, BPD also manifests in chaotic interpersonal relationships, unstable identity, suicide attempts, self-mutilation, and other selfdestructive behaviors (Skodol et al., 2002). The prevalence of BPD is $1-2 \%$ in the community, $10 \%$ in outpatient psychiatric clinics, and $20 \%$ in inpatient psychiatric settings (Gross et al., 2002; Lenzenweger, Loranger, Korfine, \& Neff, 1997; Torgersen, Kringlen, \& Cramer, 2001). Although women present with BPD more often than men in clinical settings (Skodol \& Bender, 2003), gender differences in prevalence have not been observed in population-based epidemiological

This research was supported by NIMH Grants MH-49191 and MH-36971 and by National Institute on Drug Abuse Grant DA-03188.

Address correspondence and reprint requests to: Thomas N. Crawford, Children in the Community Study, New York State Psychiatric Institute, 100 Haven Avenue, Suite 31F, New York, NY 10032; E-mail: crawfor@pi.cpmc. columbia.edu research (Torgersen et al., 2001; Winograd, Cohen, \& Chen, 2008). Approximately $3 \%$ to $10 \%$ of patients with BPD commit suicide (McGlashan, 1986; Paris, Brown, \& Nowlis, 1987; Stone, Hurt, \& Stone, 1987), thus making it a serious concern for the public health.

Despite widespread interest in BPD, relatively little empirical research has investigated its course and developmental origins in prospective data first gathered in childhood (Cohen, 2008). Longitudinal data from a community sample show that average symptom levels are highest in early adolescence and then decline over time (Cohen et al., 2008; Johnson, Cohen, Kasen, Skodol, \& Brook, 2000), probably because of maturation and socialization effects. Symptom levels in adult patients in clinical settings also tend to decline over time (Shea et al., 2002). Given parallel findings in community and clinical data, it is important to identify childhood risks that lead to early elevations in BPD symptoms but also to clarify developmental processes that delay or otherwise interfere with their usual developmental decline.

The limited data on etiology show that BPD is associated with childhood histories of abuse, 
neglect, or other relationship-related risks reported by clinical patients (Links, Steiner, Offord, \& Eppel, 1988; Zanarini et al., 2002). People in the community with elevated BPD symptoms often come from families with lower socioeconomic status (SES); those exposed to serious life events and problematic parenting also have higher risk for BPD (Cohen et al., 2008). In absence of more empirical data, theoretical models have hypothesized that childhood temperament may predispose young people to the affective instability, impulsivity, and aggression associated with BPD (Siever \& Davis, 1991). Other models hypothesize that relational factors such as insecure parentchild attachment increase risk for BPD (Agrawal, Gunderson, Holmes, \& Lyons-Ruth, 2004). The present study investigates whether BPD is associated with extended maternal separations before age 5 based on expectations that they have detrimental effects on parent-child attachment or reflect or cause other problems in the parent-child relationship.

Bowlby (1969, 1973, 1980) identified early separations as significant threats to emotional development while working in a pediatric hospital during World War II. He observed how infants were severely traumatized when separated from mother, first calling out frantically and then eventually falling into long periods of despair. Bowlby hypothesized that extended separations undermine the emotional security infants or toddlers normally experience when they are closely attached to primary caregivers. Young children rely heavily on caregivers to be available, sensitive, and responsive to their needs, especially insofar as their own coping resources are developmentally immature. As such, separations from a parent are not just alarming in early childhood; that distress is compounded when infants and toddlers have limited ability to modulate potentially overwhelming emotions on their own. Subsequent research confirmed that childhood separations are associated with lasting attachment insecurity (Moss, Cyr, Bureau, Tarabulsy, \& Dubois-Comtois, 2005; Waters, Merrick, Treboux, Crowell, $\&$ Albersheim, 2000). The link between insecure attachment and early separation is important, in turn, because insecure attachment is strongly associated with a wide range of psychopathology in young children, adolescents, and adults (see Mikulincer \& Shaver, 2007, for a review).
Insecure attachment plays an especially salient role in BPD (Agrawal et al., 2004; Bartholomew, Kwong, \& Hart, 2001). As detailed in a recent review (Levy, 2005), there is a robust link between BPD diagnoses and insecurely attached adults identified with the Adult Attachment Interview (AAI; George, Kaplan, \& Main, 1985), which elicits thoughts, feelings, and memories about childhood attachment experiences with caregivers. BPD is also robustly associated with a different domain of attachment insecurity measured with self-report questionnaires assessing close relationships between adolescents or adults (Brennan \& Shaver, 1998). However attachment insecurity is conceptualized or measured, it is closely linked with BPD and may even mediate associations between extended separations in childhood and subsequent personality disorder in adults. Attachment insecurity in adolescence and early adulthood also could maintain BPD symptoms at elevated levels during development when these symptoms normally subside.

Bradley (1979) investigated childhood histories of separation from mother before age 10 in child and adolescent patients $(n=14)$ diagnosed with BPD and compared them with various control samples. Separations before age 5 occurred more frequently among BPD patients than they did among psychotic, nonpsychotic psychiatric, and nonpsychiatrically referred delinquent youth. In contrast, there were no differences in number of separations between ages 5 and 10 recorded for BPD patients and the remaining groups. Subsequent studies investigating adult patients found significant associations between BPD and childhood histories of separation (Bandelow et al., 2005; Links et al., 1988; Reich \& Zanarini, 2001; Soloff \& Millward, 1983). Data on early separations (parental divorce, childhood hospitalizations, parental hospitalization) were drawn from chart reviews, retrospective self-reports from patients, or in some cases from family informants. When parental death was used to operationalize early separations, it was not associated with BPD in adults (e.g., Brennan \& Shaver, 1998; Paris, Zweig, \& Gruzder, 1994).

Most of the available literature is limited by reliance on retrospective self-reports by adults, typically patients from clinical samples. First, childhood amnesia prevents adult recall of experi- 
ences before age 4 or 5 , thereby raising questions about childhood risks before that age. Second, retrospectively gathered data are often distorted by memory and reporting biases (Maughan \& Rutter, 1997). Adults in emotional distress may be more ready and willing to report earlier childhood adversity; those who are functioning relatively well often underreport it instead (Maughan, Pickles, \& Quinton, 1995; Robins et al., 1985). This reporting bias may artificially increase the association between early risks and adult outcomes.

Because developmental findings are more reliable when based on repeated assessments over a range of ages, the present study investigated the association between early separations and BPD symptom trajectories in 766 youths from the Children in the Community (CIC) Study (Cohen, Crawford, Johnson, \& Kasen, 2005). This ongoing longitudinal study has tracked the course of childhood and adolescent psychiatric disorders and a wide array of developmental risks for over 30 years. Mothers provided initial data on early separations in 1975 when children were on average 5 years old. Although reported retrospectively, the interval between childhood separation and maternal reports was far less than the longer intervals associated with self-report data typically gathered from adults. In addition, mother reports are preferable to self-reports because they are not subject to childhood amnesia that limits longterm recall in adults. BPD and other psychiatric disorders were recorded in follow-up assessments of the children 8 years later and at three subsequent data intervals over the next 20 years. The present study focuses on separations lasting 1 month or longer before the child's fifth birthday to determine if they predict the course of BPD during adolescence and adulthood.

Despite its conceptual relevance, parentchild attachment security could not be evaluated in this study because it was not measured in 1975 when the sample was already between the ages of 1 and 10, and data came only from maternal interviews. However, self-reported attachment insecurity was measured at subsequent data intervals, thus making it possible to examine its association with the trajectory of BPD symptoms and to determine if it mediates the effects of early separation. Although the self-reported data used here pertain to insecure attachment in adolescence and adulthood more than early childhood (for differences in how insecure attachment has been conceptualized and measured, see Belsky, 2002; Shaver \& Mikulincer, 2002), they were tested primarily to determine if they maintain elevations in BPD symptoms over time.

From a developmental psychopathology perspective (Cicchetti \& Cohen, 2006a, 2006b; Rutter \& Sroufe, 2000), it is important to evaluate early separations in the context of other childhood risks. For instance, BPD may be associated with genetically mediated differences in temperament, especially differences in affect dysregulation and impulsive aggression (Siever \& Davis, 1991). Heritable vulnerabilities sometimes interact with childhood adversity, thereby making some people more reactive to early stressors than others exposed to the same risks (e.g. Caspi et al., 2003; Fox et al., 2005; Kaufman et al., 2004, 2006). Research on infant attachment has recently shown that heritable differences in the serotonin transporter gene increased risk for attachment insecurity, but only when combined with unresponsive or insensitive caregiving (Barry, Kochanska, \& Philibert, 2008). In research on rhesus monkeys, variations in the serotonin transporter gene interacted with early separations from mothers in ways that increased fearful and aggressive behavior in monkeys (Suomi, 2006). Although genetic data are not as yet available for this sample, constitutional differences reflected in childhood temperament may mediate or moderate long-term associations between early separation and BPD symptoms from adolescence to adulthood.

Other family risks could render children more vulnerable to lasting effects of early separation. Childhood histories of abuse and neglect are linked with insecure attachment and BPD (Stalker \& Davies, 1995; Zanarini et al., 2002). At present, it is unknown whether effects of these developmental risks combine in a simple additive manner or interact in more complex ways to increase risk for BPD. Accordingly, we examine the potential impact of later assessed risks on long-term associations of early separation and other potential early risks for BPD. These include maternal characteristics and behavior reflected in interpersonal difficulties and marital conflict, maternal consistency in childrearing practices, 
and mother's overall satisfaction with the child. Inconsistent parenting was selected based on its empirical association with BPD (Bezirganian, Cohen, \& Brook, 1993; Cohen et al., 2008). Maternal interpersonal difficulties (having trouble feeling close, blaming others, getting angry with others) was selected for its overlap with borderline symptoms, and thus a potential genetic or modeling effect on offspring. Insofar as parents may provide a dysfunctional model of interpersonal relationships, an essential aspect of BPD, we also tested whether marital conflict would predict BPD and potentially mediate its association with early separation. Maternal dissatisfaction with the child reflects both potentially harsh assessment and ways in which a mother may distance herself from her child. In addition to examining the potential influence of these variables on the BPD symptom trajectory, these variables were tested to determine if they reinforce or even potentiate effects of early separations.

Finally, we examined the impact of comorbid schizotypal personality disorder symptoms on the overall trajectory model because of known correlation and comorbidity with BPD (Cohen et al., 2008; Kavoussi \& Siever, 1992) and recent findings linking it with early separations in this cohort (Anglin, Cohen, \& Chen, 2008). Rather than looking for mediating or moderating effects, this variable was included to rule out the possibility that it might explain an association found between early separation and BPD.

\section{Improving Our Understanding of Developmental Data}

Here we present data on factors affecting the developmental trajectory of BPD symptoms assessed four times over two decades, beginning with early childhood risks. It is worth presenting our view of an important aspect of such data presentation that is not often present, we admit, previously not even always in the data from the cohort study on which this investigation of development is based. Of course, much developmental research is cross-sectional, perhaps with retrospective reports or other data about past exposures to experiences that are likely to have an influence on the measure on which the current focus is being placed. When data are collected repeatedly, the most common number of times is two, often within a single school year, or over 2 consecutive years. Nevertheless, the issue we address remains: it is informative to present data in such a way that the magnitude of developmental changes in measured data is understandable, and the magnitude of other influences on these variables, including on their developmental changes, can similarly be understood in a common frame.

There are two complications with this otherwise easily accomplished goal: one is problems with the reliability of the variables included in the developmental trajectories and of the predictors of potential influences on the developmental path. Estimates of the "true" or valid aspects or influences of many of the scaled variables we assess require multiple measures of the component constructs, latent variable techniques, and valid interpretations of the meaning of changes in coefficients. For multiple time point measures these can get quite complicated, but are very useful when assumptions can be met. Such methods are almost never used for more than two or three time points, to our knowledge. Those of us who have been using multiple time-point assessments have gradually realized a delicious surprise: any kind of multiple assessments of a variable, including those taking place over a number of years, improve the reliability and probable validity of their overall average and, given more than two assessments, of their trajectory. In other words, problems of reliability/validity of a trajectory of such repeated assessments are diminished, probably substantially, relative to single assessments.

The other problem is how to scale variables so that there can be a common understanding of the magnitude of impact of a given influence on a developmental trajectory over two or more assessments. An elegant and familiar solution, if slightly more complicated than coping with a single variable, is standardization across the trajectory. This is accomplished by the following simple process. First, a preparation for an analysis of a trajectory requires "stacking" of the data, so that for each unit being studied (usually a person with a particular identifying number) the data are rearranged so that every assessment of a given variable (each measure that is remeasured over time) for each ID is in a single column of data. Which assessment each variable value comes from is identified by some other 
time-indicating variable, stacked in parallel (e.g., age, date, wave of data assessment). Any "timevarying" predictors to be employed in the analysis may also be stacked in the file.

The next step is quick and easy: simply standardize the column of data that includes the entire set of measures of your dependent variable. Second, when your main interest is developmental change, center your measure of age (or time) at the mean value across the trajectory data. In consequence, there will be two large advantages: first, the magnitude of the rate of developmental change in the dependent variable will be easily understood. Whether the time units are dates, ages, or waves, analyses using this variable as a trajectory predictor will estimate the mean developmental change per time unit expressed in standard deviations. Second, the estimated effects of all predictors can be separated into effects on the overall level estimated at the average age and effect on the rate of change, estimated by the product of the predictor and the time variable (e.g., age). More complex analyses can investigate nonlinear developmental changes, for which appropriate transformations of the time variable may be complex.

Having accomplished this transformation, additional understanding of effect magnitudes will come by standardizing predictors that are not scaled in understood units, such as a number of something, or no or yes. Doing so will enable comparison of the effects of different measures on the trajectory mean, and better understanding of the magnitude of potential mediation effects when variables are combined in trajectory-predicting equations.

In this paper our focus will naturally fall on the substantive issues, but we will try throughout to make clear our interpretation of the magnitude of significant effects as assessed, in keeping with our belief that we must ultimately know not only what influences development, but also accumulate evidence about magnitudes of such influences.

\section{Method}

\section{Participants}

The CIC sample is a cohort of children originally drawn in 1975 from randomly sampled family households in rural, suburban, city, and central city areas in two counties in upstate
New York, picked for demographic resemblance to the full US population (prior to large-scale immigration from Latin America). The initial interviews of 976 mothers related to one child between ages 1 and 10 randomly selected from age-eligible children in the household. Interviews covered a wide array of issues related to offspring well-being including the child's health, temperament, attitudes, behavior, relationship with parents, and social environment, including parental problems, and significant events such as extended separations, divorce, and deaths of family members. Eighty-five percent of the original families were identified and relocated in 1983 , and $78 \%$ agreed to participate in a follow-up interview with the mother and/or a separate interview with the sample child. Many families living in poor urban areas could not be located because whole neighborhoods had been torn down for urban renewal. To replace these young, urban, and poor families excessively lost to follow-up, this sample was supplemented with 54 families from comparable areas of urban poverty within the sampled region who had children between ages 9 and 12 years. The sample's racial distribution (91\% Caucasian, $8 \%$ African American) and SES (21\% with family income below the poverty line at some time in childhood, $25 \%$ with upper middle class educational and income) were representative of the two counties.

Data for the present study were drawn from 766 youth who participated in two or more follow-up interviews conducted in $1983(N=756$; mean age $=13.7, S D=2.6$ years $), 1985-1986$ $(N=746$; mean age $=16.1, S D=2.8$ years $)$, 1991-1993 $(N=749$ : 717 with complete information; mean age $=22.0, S D=2.7$ years $)$, and 2001-2004 $(N=678$; mean age $=33.1, S D=$ 2.8 years). After maintaining youth retention rates exceeding 90\% through the 1991-1993 assessment, participation dropped to about $84 \%$ in the 2001-2004 assessment. Similar problems of attrition have been observed in other recent longitudinal studies of community and clinical samples and reflect loss because of unknown relocation and failure to respond to study communications. Men surveyed in adolescence have been less likely than women to participate in adulthood, although there have been very few respondents who have declined to continue in the study. 
Race, SES, and psychiatric disorder have not been related to attrition.

This cohort study includes participants born over a full decade. In consequence at every age between 9 and 39 a number of assessments were available for these analyses, easing concerns about potential nonlinear changes that might have taken place in the years between assessments. Additional details about this community sample are available on the study Website (http://nyspi. org/childcom). Informed consent or assent was obtained for all interviews and a NIH Certificate of Confidentiality exists for these data.

\section{Measures}

$B P D$. There were no existing measures of Diagnostic and Statistical Manual of Mental Disorders $(D S M)$ personality disorders for adolescents when this cohort was first followed up in 1983. Items were drawn from the Personality Diagnostic Questionnaire (Hyler, Rieder, Spitzer, \& Williams, 1982; Hyler, Skodol, Kellman, Oldham, \& Rosnick, 1990) and adapted as necessary to make them age appropriate. Additional items have been selected from the CIC protocol to produce measures that closely correspond to DSMIV (American Psychiatric Association, 1994) diagnostic criteria. The present report used BPD symptom scales based on 15 youth-reported items assessed at mean ages 13.7, 16.1, 22.0, and 33.1 (mean Cronbach $\alpha=.74$ ). ${ }^{1}$ Items in the BPD scale (measured on Likert scales mostly ranging from 1 to 4 ) were summed and standardized as described above, thus making developmental change easier to interpret. Convergent

1. Earlier published reports on personality disorders in this cohort have employed combined maternal-child reported symptoms using DSM-III (American Psychiatric Association, 1980) and DSM-IV criteria. Evidence based on the longitudinal data suggests that youth self-report may predict prognosis and dysfunction better, and recent reports have employed these measures. We have published data elsewhere on a 22-item self-report BPD scale (Crawford et al., 2005), which used 7 additional items available only in the last two data waves. The abbreviated 15-item scale was used here because it provides a consistent repeated measure at all four data intervals from adolescence into adulthood, including the adolescent assessments at mean ages 13.7 and 16.3. The correlation between the abbreviated and complete BPD scale is .97 . validity for the BPD scale at mean age 33 is good; it is positively correlated with the BPD selfreport scale on the Structured Clinical Interview for DSM-IV (SCID-II; First, Gibbon, Spitzer, Williams, \& Benjamin, 1997) Personality Questionnaire $(r=.74, p<.001)$. By comparison, the median correlation between self-report BPD scales was only .41 when agreement was assessed in 13 other studies (Modestin, Ern, \& Oberson, 1998). The BPD scale was stable across 11 years from mean ages 22 to $33(r=.51, p<$ .01 ), and predicted a clinician's assessment of global functioning after completion of a clinical interview (Crawford et al., 2005). This measure has been validated in additional longitudinal research showing that adolescent BPD is associated with adult borderline symptoms and diagnoses, lower academic and occupational attainment, higher need for services at mean age 33 (Winograd et al., 2008), higher partner conflict (Chen et al., 2004), and long-term reductions in quality of life (Chen et al., 2006).

Maternal separation. Mothers provided data about early separations in 1975 and at follow-up in 1983. Mothers were initially asked, "Have there been any times since (CHILD) was born that you have been separated more than a month either because you were away or because (CHILD) was away from home?" In 1983 mothers were asked, "How many times since 1975 have you been separated from (CHILD) for more than a month?" In both cases positive responses were followed up with questions about the duration, timing, and reason for the separation. Based on Bradley's (1979) finding that early separations increased risk for BPD, we identified participants who were separated from mother for at least one month before age 5. This age was also selected as a reasonable cutoff because maternal separations become more normative after age 5 (e.g., enrollment in primary education, going away to summer camp, etc.). Altogether, 35 children (4\% of the sample) were exposed to this operational definition of early separations. Five children were separated because of childhood hospitalization or illness, 13 had extended separations because of maternal hospitalization or illness, and 17 were separated for other reasons. These other separations included extended visits to a relative, or the mother's departure for personal, educational, or professional 
reasons. We used the length of separations (mean $=15.7$ weeks, range $=4-59$ weeks) as a scaled measure for statistical analyses.

No early separations were attributable to foster care placement. To determine if early parental death increased risk for BPD, we identified 11 children whose mother or father died before age 5. However, early parental death was not associated with BPD in any statistical analyses. Parental divorces that occurred before the child turned 10 were also not predictive of BPD symptoms in the offspring. Based on these findings, we did not include parental death or divorce in this paper.

Demographic factors. SES was measured as a standardized sum of standardized measures of years of maternal and paternal education, occupational status, and family income. SES varies inversely with BPD symptoms (Cohen et al., 2008). Gender and race (White vs. not White) were not associated with BPD in this community sample. Despite higher rates of women in BPD clinical populations, recent evidence strongly suggests that this differential may be because of biases in diagnosing practice (Skodol \& Bender, 2003).

Child risk factors. Mothers were asked about their child's temperament in 1975 using items adapted from the Thomas and Chess longitudinal study that tracked temperament from childhood to adolescence (Chess \& Thomas, 1978; Thomas \& Chess, 1977; Thomas, Chess, $\&$ Birch, 1970). Although items varied by age, a fixed set of items covered ages 4 to 10. Eight years later newly recruited mothers with children ages 9 to 12 and mothers of children from the original sample who were then 9 to 12 were administered the same items. To minimize age effects, the assessment closest to age 9 was selected for each child and then adjusted for sex-specific age changes, converting the data covering ages 6 to 12 to estimates at a constant age 9. Two temperament scales were selected as predictors based on correlations with early separation. The "crying/demanding" scale uses seven items (Cronbach $\alpha=.68$ ) to assess frequency of crying, mood reactivity, and demands for attention. Two additional items in the scale asked whether the child's crying annoyed mother and father. The "crying/demand- ing" scale corresponds to early measures of "difficult" temperament (Thomas \& Chess, 1977; Thomas, Chess, \& Korn, 1982). The "angry/tantrum" scale uses four items $(\alpha=.47)$ to assess how frequently the child cries or complains loudly, screams when angry, kicks and throws things, and has temper tantrums. These items resemble those in the "frustration" scales of the Children's Behavior Questionnaire (Rothbart, Ahadi, Hershey, \& Fisher, 2001).

Maternal risk factors. Maternal interpersonal difficulties, marital conflict, inconsistency in child rearing, and dissatisfaction with the child were reported by mothers when children were on average 13.7 years old (age range $=9.1$ 18.6 years). Maternal interpersonal difficulties were measured with five items ( $\alpha=.73$ ) adapted from the System Checklist-90 (Derogatis, Lipman, Rickles, Uhlenhuth, \& Covi, 1974) that assess problems getting close to others, having temper outbursts, feeling tense or anxious, feeling easily annoyed, and blaming others. The Marital Conflict Scale is a three-item index ( $\alpha$ $=.58$ ) that assesses three components Fincham and Osborne (1993) identified as important subdimensions of marital conflict: (a) the frequency, (b) their average intensity (calm to potential violence), and (c) whether the differences end up in negotiated agreement or ongoing disagreement. Marital conflict has been linked to problematic offspring emotional security (Davies \& Cummings, 1994), a component of BPD symptoms.

Maternal inconsistency ( $\alpha=.63$ ) was measured with three mother-reported items adapted from a maternal inconsistency scale (Schaefer, 1965) and two items from a separate rule scale (Jessor, Graves, Hanson, \& Jessor, 1968). Sample items include "How consistent are you in the way you handle your child?" and "I often change the rules that my child is supposed to follow." Maternal dissatisfaction was measured with six original items $(\alpha=.81)$ that assess maternal satisfaction/ dissatisfaction with the child's physical appearance, intellectual and interpersonal ability, moral and emotional behavior, and achievement in school. Prior research has supported the validity and predictive utility of these three measures (Berenson, Crawford, Cohen, \& Brook, 2005; Bezirganian et al., 1993; Cohen, 1996; Cohen \& Cohen, 1996; Crawford, Cohen, Midlarsky, \& 
Brook, 2001; Johnson, Cohen, Kasen, Ehrensaft, \& Crawford, 2006; Schaefer, 1965).

Child maltreatment. Official records identified 37 participants who met legal criteria for sexual abuse, physical abuse, or neglect.

Insecure attachment. Self-reported attachment insecurity was assessed at mean age 16.1 with the CIC attachment scales (Crawford et al., 2006) using 13 items ( $\alpha=.87)$ to measure attachment anxiety (fears of abandonment) and 14 items ( $\alpha$ $=.87$ ) to measure attachment avoidance (discomfort with intimacy). These scales assess the same attachment dimensions as the Experience in Close Relationships Inventory (ECR; Brennan, Clark, \& Shaver, 1998), a widely used instrument first published after adolescents in the CIC study were already interviewed. Item content in these scales focused on how respondents felt about their closest relationships at the time of assessment. Correlations between anxious attachment scales on CIC and ECR measures $(r=.70)$ and associations between corresponding avoidant attachment scales $(r=.71)$ were reasonably high in a separate college validation sample $(N=307)$, thus supporting the convergent validity of these scales.

Additional risks. Schizotypal personality disorder symptoms correlate with BPD symptoms (Cohen et al., 2008) and have recently been linked with early separations (Anglin et al., 2008). Accordingly, schizotypal symptoms assessed in early adolescence were examined to control for their potentially confounding effect on risks for BPD, especially early separation. Schizotypal symptoms were measured with self-report items from the CIC protocol that match DSM diagnostic criteria (Crawford et al., 2005). The internal consistency of this 16-item scale was acceptable (mean $\alpha=$ $.61)$. The same scale correlated .49 with the Schizotypal Scale on the Structured Clinical Interval for DSM-III-R (SCID-II) Personality Questionnaire and -.46 with clinician reported Global Assessment of Functioning Scale (Crawford et al., 2005).

\section{Data analyses}

Hierarchical linear regression modeling (HLM) was used to investigate associations between early separations and age trajectories for BPD symptoms from age 9 to 39. The SAS PROC MIXED program generated maximum likelihood estimates of fixed effects (average) and random effects (differences between participants) associated with each study variable. Linear trajectories were assessed in all HLM models represented by the age variable. Age was centered at age 20: here the $(\sim 0)$ intercept equals the mean level of BPD symptoms at age 20 when all other predictors equal zero.

Initial analyses examined the effects of early separation on trajectory of BPD symptoms as well as the reasons given for those separations. Subsequent analyses added the covariates specified above to determine if they mediate, moderate, or confound the effects of early separations on subsequent BPD symptoms. To facilitate comparisons of effect sizes across models, all scaled predictors (except weeks of separation and age) were standardized. (For interested readers, equations used in HLM analyses are available from the author upon request.)

\section{Results}

HLM models confirmed that extended separations from mother before age 5 were associated with BPD symptoms in adolescence and adulthood. Table 1 shows that children with early separations had significantly higher BPD symptoms, 0.0172 $S D /$ week of separation, than children not separated from mother. At the average separation duration of 15.7 weeks, we find the effect $=15.7 \times$ $0.0172=0.27 S D$, a surprisingly large effect considering the elapsed time between the early childhood risk and the average age 20 when BPD symptoms were assessed. As expected, BPD symptoms decreased with age $(-0.0341 S D /$ year), thus reflecting normal declines observed across adolescence and early adulthood. However, the level of BPD symptoms declined less over the full trajectory for those who had extended early separations (see Figure 1 for the estimate for the average 15.7-week separation). On average, young people from families with lower SES had significantly higher risk for BPD.

Dummy variables were used to parse the separation variable into three independent variables based on reasons why mother and child were separated: mother's illness $(n=13)$, 
Table 1. Early childhood separations from mother (up to age 5) predict borderline personality symptoms across adolescence and adulthood

\begin{tabular}{|c|c|c|c|}
\hline & Estimate & $S E$ & $\begin{array}{c}\text { Est. Aver. } \\
\text { 15.7-Week Sep. } \\
\text { Duration Effect }\end{array}$ \\
\hline \multicolumn{4}{|c|}{ Early Separation and Demographic Risks } \\
\hline Standardized BPD symptom change/year & $-0.0341 * * *$ & 0.0021 & \\
\hline SES & $-0.1540 * * *$ & 0.0261 & \\
\hline Separation (weeks) & $0.0172 * *$ & 0.0065 & 0.270 \\
\hline Separation $\times$ symptom change & $0.0013 * *$ & 0.0005 & $60 \%$ reduction \\
\hline \multicolumn{4}{|c|}{ Evaluating Different Reasons for Separation } \\
\hline Standardized BPD symptom change/year & $-0.0339 * * *$ & 0.0020 & \\
\hline SES & $-0.1621^{* * *}$ & 0.0260 & \\
\hline Separation due to mother's illness (weeks) & -0.0222 & 0.0171 & \\
\hline Separation due to child's illness (weeks) & -0.0493 & 0.0741 & \\
\hline Separation for other reasons (weeks) & $0.0179^{*}$ & 0.0077 & \\
\hline $\begin{array}{l}\text { Separation for other reasons } \times \text { symptom } \\
\text { change }\end{array}$ & $0.0022 * * *$ & 0.0005 & \\
\hline
\end{tabular}

Note: BPD, borderline personality disorder; SES, socioeconomic status.

$* p<.05 . * * p<.01 . * * * p<.001$.

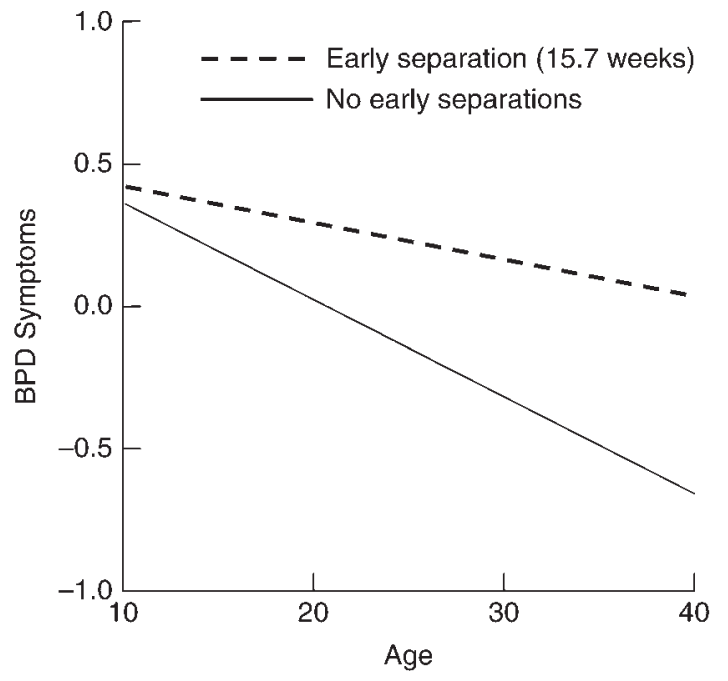

Figure 1. Early maternal separations increase long-term risk for borderline personality symptoms (standard deviations).

child's illness $(n=5)$, or other reasons $(n=$ 17). These subgroups were compared with children who did not experience extended separations before age 5. Separations for other reasons (extended visits to a relative, a mother's departure for personal or professional reasons) predicted higher BPD symptoms (0.0179 SD for each week of separation) as well as slower developmental declines in symptoms. Separations attributed to mother's illness did not significantly predict BPD symptoms. Separations stemming from childhood illness initially seemed associated with subsequent risk for BPD. However, closer examination revealed that four respondents 
had hospitalizations that were similar in duration (4-6 weeks) and one had a much longer hospital stay (26 weeks). When this outlier was removed, the association between BPD and separations attributed to childhood illness disappeared. To avoid reporting unreliable results, findings in Table 1 were based on the sample without the single outlier. Nevertheless, because these analyses of cause-specific separation may have missed true associations with later BPD symptoms because of very small numbers of children in subgroups, we carried out subsequent HLM analyses for the full set of children who experienced extended separation from their mothers regardless of reason. We employed weeks of separation as the predictor in all subsequent analyses.

Table 2 reports analyses that examined the cumulating and mediating effects of other risks, entered in the sequence that they took place or were first measured. Thus, we can examine the mediation of the association of early risks, including early separation, on the developmental trajectory of BPD symptoms by later risks.

The baseline model specified childhood separations as a predictor of both mean and slope (linear change with age) of BPD symptom trajectory. Overall estimates of the effects of early separation were calculated by multiplying the estimated effect per week by the mean separation duration $=$ 15.7 weeks (reported in the third column of Table 2). Estimates of the effects on BPD symptom change per year are similarly made by multiplying the weekly effect by the mean number of weeks and dividing this estimate by the reference annual mean change from the same equation.

Subsequent models identify later trajectory predictors and enable estimation of potential meditational effects of these predictors on the association between early separation and the BPD symptom trajectory. Moderating effects of later risks on the early separation effects were tested by adding products of these variables to the prediction equations, but none were statistically significant. We also did not find a later risk that was related to the slope (change per year) of the trajectory of BPD symptoms, and therefore, these are not included in the table.

In the baseline model, the average effect of early separations (mean $=15.7$ weeks) was associated with increases in BPD symptoms that were over $0.25 S D$ in magnitude, a rather sub- stantial effect over a 15 - to 19 -year period. In the baseline model we see that annual symptom decline is estimated to be $65 \%$ slower for those at the average number of separated weeks.

Model 1 in Table 2 adds child temperament risks estimated at age 9. Each standard deviation increase in angry temperament was associated with a 0.0951 SD increase in BPD symptoms. Each standard deviation increase in crying/demanding temperament was independently associated with a $0.1054 S D$ increase. Parameter estimates for differences in symptom mean and symptom trajectory associated with early separation changed little when temperament risks were added to the model. Thus, maternal reports of child temperament did not mediate early separation effects on BPD, indicating independence of these risks.

Model 2 shows that a documented history of abuse was associated with a $0.5603 S D$ increase in risk for BPD symptoms, an estimate consistent with previous evidence of the history of abuse reported by BPD patients. However, abuse did not substantially mediate the early separation effects on the BPD symptom trajectory.

Model 3 adds parental variables to the potential predictors, all assessed at the same time as the first offspring report of BPD symptoms (mean age $=13.7$ ). We see that inconsistent mothering and low maternal satisfaction with the child were significant predictors of higher BPD symptoms. For each standard deviation decrease in maternal satisfaction, there was a $0.1579 S D$ increase in BPD symptoms. Inconsistent mothering had a much smaller independent effect. These mother-reported variables also partially mediated the effects of her earlier reports of her child's temperament. The independent effect of crying-demanding was no longer statistically significant, and the effect of angry temperament was lowered almost $40 \%$. Maternal interpersonal difficulties and parental conflict did not significantly predict offspring BPD symptoms independently of other variables in the model.

Model 4 adds anxious and avoidant attachment measured in middle adolescence. For each standard deviation increase in attachment anxiety, there was a notably large elevation in BPD symptoms over the trajectory $(0.3756 S D)$ : independent effects of attachment avoidance 
Table 2. Estimated standardized effects of risks on the trajectory of borderline disorder symptoms from early adolescence to 20 years later as adults

Est. Aver.

Estimate $\quad S E \quad$ Duration Effect

\begin{tabular}{lccc}
\hline \multicolumn{4}{c}{ Baseline Comparison Model } \\
\hline BPD symptom change/year & $-0.0341^{* * *}$ & 0.0021 & \\
Separation (weeks) & $0.0201^{* *}$ & 0.0067 & $0.270 S D$ \\
Separation $\times$ symptom change & $0.0014^{* *}$ & 0.0005 & $65 \%$ reduction \\
\hline \multicolumn{4}{c}{ Model 1: Separation and Childhood Temperament Risks } \\
\hline BPD symptom change/year & $-0.0340^{* * *}$ & 0.0021 & \\
Separation (weeks) & $0.0157^{*}$ & 0.0065 & $0.246 S^{*}$ \\
Separation $\times$ symptom change & $0.0014^{* *}$ & 0.0005 & $65 \%$ reduction \\
Angry temperament (age 9) & $0.0951^{* *}$ & 0.0284 & \\
Crying/demanding (age 9) & $0.1054^{* *}$ & 0.0289 & \\
\hline
\end{tabular}

Model 2: Adding Abuse History

\begin{tabular}{lccc}
\hline BPD symptom change/year & $-0.0339^{* * *}$ & 0.0021 & \\
Separation (weeks) & $0.0130^{*}$ & 0.0064 & $0.204 \mathrm{SD}^{*}$ \\
Separation $\times$ symptom change & $0.0014^{* *}$ & 0.0005 & $65 \%$ reduction \\
Angry temperament age 9 & $0.0970^{* * *}$ & 0.0280 & \\
Crying/demanding age 9 & $0.0951^{* * *}$ & 0.0285 & \\
Abuse record & $0.5603^{* * *}$ & 0.1149 & \\
\hline
\end{tabular}

Model 3: Adding Maternal Risk and Parenting

\begin{tabular}{lllc}
\hline BPD symptom change/year & $-0.0340^{* * *}$ & 0.0209 & \\
Separation (weeks) & 0.0106 & 0.0062 & 0.166 SD \\
Separation $\times$ symptom change & $0.0014^{* *}$ & 0.0005 & $65 \%$ reduction* \\
Angry temperament age 9 & $0.0597^{*}$ & 0.0276 & \\
Crying/demanding age 9 & 0.0500 & 0.0288 & \\
Abuse & $0.5131^{* * *}$ & 0.1127 & \\
Parental conflict & 0.0000 & 0.0274 & \\
Maternal interpersonal difficulty & 0.0365 & 0.0302 & \\
Inconsistent mothering & $0.0555^{*}$ & 0.0273 & \\
Maternal satisfaction with child & $-0.1579^{* * *}$ & 0.0298 & \\
\hline
\end{tabular}

Model 4: Adding Adolescent Attachment

\begin{tabular}{lllc}
\hline BPD symptom change/year & $-0.0340^{* * *}$ & 0.0020 & \\
Separation (weeks) & 0.0079 & 0.0053 & $0.141 \mathrm{SD}$ \\
Separation $\times$ symptom change & $0.0015^{*}$ & 0.0005 & 69\% reduction* \\
Angry temperament age 9 & $0.0606^{* *}$ & 0.0235 & \\
Crying/demanding age 9 & 0.0294 & 0.0245 & \\
Abuse & $0.4249^{* * *}$ & 0.0959 & \\
Inconsistent mothering & 0.0236 & 0.0232 & \\
Maternal satisfaction with child & $-0.1313^{* * *}$ & 0.0253 & \\
Anxious attachment in adolescence & $0.3756^{* * *}$ & 0.0225 & \\
Avoidant attachment in adolescence & $0.0934^{* * *}$ & 0.0220 & \\
\hline \hline
\end{tabular}

Note: BPD, borderline personality disorder.

$* p<.05 . * * p<.01 . * * * p<.001$. 
were also significant but much less dramatic $(0.0934 S D)$. In this model the (modest) estimated effects of maternal inconsistency were no longer statistically significant, and nearly $20 \%$ of the association of abuse history was mediated by these variables. However, the effect of early separation on the lesser decline of BPD symptoms with age remained robust, and is nearly $70 \%$ less per year than estimated in those participants without these risks.

In separate models (not shown in Table 2) we added schizotypal PD symptoms assessed in the initial assessment (mean age $=13.7$ ) to these predictors. This inclusion did not affect the estimates of early separation effects on the BPD symptom trajectory, and only the effect of avoidant attachment in adolescence was changed from marginal to not significant in this model. Schizotypal symptoms are not viewed as an unambiguous "mediator" because the causal direction between symptoms of these two disorders is not known. However, we also evaluated the trajectory of schizotypal symptoms (measured as a time-varying covariate from early adolescence to middle adulthood) to determine if its own associations with early separations (Anglin et al., 2008) and co-occurring BPD symptoms (Cohen et al., 2008) might account for the effects observed here. The estimated effect of early separation on the trajectory of BPD symptoms did not change when co-occurring schizotypal symptoms were added to the model.

\section{Discussion}

Associations between early childhood risks and adult psychopathology are often hypothesized but seldom observed. This outcome stems not just from a shortage of longitudinal data; more substantively, it reflects how individual trajectories are affected by many factors throughout development (e.g., heritable vulnerability or resiliency factors, stability or change in family composition, exposure to new relationships outside the family, etc.) that influence whether or not early risks eventually manifest as psychopathology in adults. In this context, it is noteworthy that extended childhood separation from mother before age 5 was associated with BPD symptom trajectory up to 30 years later.

The present study reinforces prior research linking early separation and BPD in children and adolescents (Bradley, 1979). Our findings also reinforce studies that found associations between BPD in adults and early separations based on retrospectively reported data (Bandelow et al., 2005; Links et al., 1988; Reich \& Zanarini, 2001; Soloff \& Millward, 1983), especially insofar as our prospective data are less likely to be distorted by memory and reporting biases. Not only were BPD symptoms higher on average among children with early separations, the same symptoms declined at significantly slower rates than those observed in young people without early separations. In most cases, elevated personality disorder symptoms decrease in adolescence because of normative maturation and socialization processes (Cohen $\&$ Crawford, 2005). Slower declines in young people with early separations suggest that their emotional development lags behind age peers during adolescence and early adulthood.

Childhood separations by themselves may not increase long-term risk for BPD. Early separations attributed to extended child or maternal hospitalization were not associated with the BPD symptoms or their developmental trajectory. Definitive results, however, will require longitudinal data on more cases than those available here These findings contrast with those observed when 66 adult patients with BPD were compared with 109 nonpsychiatric controls (Bandelow et al., 2005). Patients had more retrospectively reported childhood hospitalizations than healthy controls $(72.7 \%$ vs. $37.6 \%, p<.001)$, although these hospitalizations were not limited to the time between birth and age 5. Adults with BPD also reported more childhood separations because of maternal hospitalizations than controls $(65.6 \%$ vs. $34.9 \%, p<.001)$. Different outcomes may stem from differences between clinical and community samples. Each childhood risk increases not just the likelihood of psychopathology; as risks and psychopathology accumulate they also increase the likelihood of treatment seeking, thereby resulting in an ascertainment bias in clinical samples that may artificially inflate associations between BPD and childhood and maternal hospitalizations. Recall biases in retrospectively reported data may also inflate associations observed by Bandelow et al. (2005).

Long-term risk for BPD in our community sample was more reliably associated with 
childhood separations attributed to other reasons: mothers leaving for personal reasons, relocating for professional or educational purposes, or children going away for extended visits to a relative. Although we lack detailed information about what motivated these separations, parents who sent children to live with relatives may have done so because of the child's behavioral problems that were difficult to tolerate or manage. In other cases, mothers may have placed a higher priority on educational, professional, or personal goals than on parenting responsibilities, at least for the duration of their absence. Extended separation thus could be a long-term risk only insofar as they reflect a lack of maternal investment in caregiving.

Such separations may be difficult for young children to understand, perhaps leading them to blame themselves for mother's absence. These cognitions may have a harmful impact on the child's mental representations of self and other: the "internal working models" emphasized in Bowlby's theory (Bowlby, 1969, 1973, 1980; Bretherton \& Munholland, 1999). If children believe that their mother did not want to be with them or care about their needs, they may form mental representations of themselves as being unworthy of love and expect significant others to be uninterested or rejecting. To the extent that such affectively laden representations undermine attachment security, they may also increase risk for psychopathology. In these analyses we find that the elevation of BPD symptoms at the trajectory mean age was about $0.25 S D$ for separations of the average length, a small to medium effect when considered over this extended period. Perhaps more impressive is the impact on the normative annual BPD symptom decline, running at about one-third the rate experienced by those without early separation. This effect was essentially unmediated by any of the measured later risks.

Maternal reports of childhood temperament predicted higher BPD symptoms at the trajectory mean, with about a $0.1 S D$ increase in symptoms per standard deviation increase. Associations between childhood temperament and BPD specifically support Siever and Davis's (1991) model of this disorder. The impulsive aggression hypothesized in their model, indexed here by angry tantrums at mean age 9 , predicted BPD symptoms in adolescence and adulthood. BPD was also associated with frequent crying, mood reactivity, and demands for attention at mean age 9, which may reflect the heritable predisposition to affective instability Siever and Davis hypothesize in their model. The childhood temperament variables mediated almost $20 \%$ of the early separation association with mean BPD symptoms over the trajectory (reduction from .0201 to .0157 per week) but did not mediate the impact of early separation on estimated annual decline in symptoms. Associations between childhood temperament and BPD are noteworthy in themselves because they extend well into adulthood and have not been examined previously in developmental data. Of course, variables measuring angry tantrums and crying/demanding behaviors could also stem from family disturbances instead of genetically mediated differences in temperament: as noted below, the effects of the temperament variables were mediated primarily by the maternal parenting risks.

A recorded history of child abuse had the expected very large association with BPD symptom mean over the trajectory (increase of $>0.5$ $S D$ for those with such a history), but very modest mediation effect on early separation relationship with this mean and none on the trajectory slope or on the temperament effects. Severe abuse has been linked with lasting disruptions of the hypothalamic-pituitary-adrenal system, which regulates the release of glucocorticoid hormones into the bloodstream in normal daily cycles but also in response to stress (Smith \& Vale, 2006; Van Voorhees \& Scarpa, 2004). Severe or prolonged stress from familial adversity in early development may result in hyperactivation of the hypothalamic-pituitary-adrenal system (hypercortisolism) and amygdala hyperfunction (fear reaction; Charmandari, Kino, Souvatzoglou, \& Chrousos, 2003; Heim \& Nemeroff, 2001; Tarullo \& Gunnar, 2006). Additional research is needed to determine if this physiological mechanism mediates associations between early risks and BPD (Garner et al., 2007; Lieb et al., 2004).

Maternal interpersonal difficulties did not relate to offspring BPD symptoms, nor did parental conflict. These findings surprised us because we anticipated that maternal behaviors reflecting 
some of the serious aspects of BPD symptoms would have presented a "model" of expected or normative behavior to her child, as conflict between the parents might also have done. They might even have reflected a component of potential genetic transmission. However, inconsistent parenting and to a much larger degree her dissatisfaction with her child as reported to a stranger (the interviewer) did predict subsequent BPD symptoms over the trajectory. These predictions mediated the effect of early separation enough to reduce the estimated independent effect on mean symptoms to a level that is no longer statistically significant. Although maternal dissatisfaction could plausibly amplify an existing risk from early separation (e.g., by reinforcing internal working models that make the child feel unworthy of love), no moderating effects were observed. In addition, these parent variables did not mediate the lesser decline in BPD symptoms with age in those who had experienced such separations.

We have long viewed very low scores on the satisfaction variable as a representation of near hostility of the mother toward the child: consistent dissatisfaction of her child's appearance, intelligence, relationships, emotional behavior, achievement, and moral behavior. We also note that, although temperament risks were not mediated by abuse records, they were substantially mediated by inconsistent mothering and maternal satisfaction with the child (both mother reported). This pattern, if accepted as measured in these data, suggests that even very problematic "difficult" children may turn out to be fairly welladjusted adults if accepted and managed wisely by parents (Thomas \& Chess, 1978).

Risk associated with early separation was independent of self-reported attachment anxiety (abandonment fears) and avoidance (discomfort with intimacy) experienced in close relationships in mid to late adolescence. Attachment anxiety in close/romantic relationships was notably more associated with higher BPD symptoms (over $0.5 S D$ of BPD symptoms for every standard deviation of anxious attachment) than was avoidant attachment (about $0.125 S D$ of BPD symptoms for every standard deviation of avoidant attachment). This high relationship is consistent with the salience of abandonment fears in BPD reported elsewhere in the literature (for a review, see Levy,
2005). The association with attachment avoidance and BPD has been more variable, sometimes showing positive associations and other times inverse associations with BPD. This variability may reflect differences in how people defend against high attachment anxiety, some by distancing themselves from close relationships (high avoidance), others by clinging to close friends or romantic partners instead (low avoidance). Some variability may also reflect differences in sample composition; on average, treatment-seeking individuals in clinical samples may experience less avoidance, and people selected at random for community samples may report higher avoidance instead.

Contrary to expectations, self-reported attachment anxiety and avoidance did not mediate the association between early separation and BPD symptoms. Indeed, the available attachment variables were not significantly associated with early separations, probably because self-report attachment questionnaires focus on close relationships between adolescents or adults, not the parent-child relationships studied in the childhood attachment literature. The estimated effects of inconsistent parenting were somewhat mediated by the adolescent attachment variables in this model.

Instead, the self-reported attachment variables reduced the estimated impact of child abuse history on BPD symptoms by about $20 \%$. To the extent that child abuse increases attachment insecurity during adolescence and adulthood, it may instill ongoing attachment anxiety that is strongly associated with BPD. If so, attachment anxiety may play a key role in maintaining elevated BPD symptoms across adolescent and early adult development when these symptoms might otherwise subside.

Child maltreatment by primary caregivers has also been linked with "disorganized attachment" (Solomon \& George, 1999), which often occurs when maltreated children seek comfort from primary caregivers who have abused them in the past. When young children fear their primary caregiver, or when the caregiver herself is afraid, children experience approach/ avoidance conflicts and often have dazed dissociative responses on reunion following a brief separation. When the effects of childhood maltreatment and disorganized attachment persist 
into adulthood, they are thought to increase risk for BPD (Fonagy, Gergely, Jurist, \& Target, 2002; Holmes, 2004; Salzman, Salzman, \& Wolfson, 1997).

Finally, the link between early separation and BPD cannot be attributed to schizotypal personality disorder symptoms, which were found to be associated with early separations in this cohort (Anglin et al., 2008). Although borderline and schizotypal PD are correlated (Cohen et al., 2008; Kavoussi \& Siever, 1992), they are nevertheless distinct forms of personality pathology (Sanislow et al., 2002). These associations between early separation and two distinct personality disorders indicate that this developmental risk is not unique to BPD. Consistent with the principal of multifinality (Cicchetti \& Rogosch, 1996), early separations may lead to developmental trajectories associated with a wider range of psychopathology.

Associations between early separations and distinct personality disorders or other forms of mental illness may reflect different underlying mechanisms. In fact, the association with the schizotypal symptom trajectory was limited to those children who had both experienced these early separations and had high angry temperament as assessed here at age 9 (Anglin et al., 2008), whereas in these data there were no identified moderating effects of other risks. Although mechanisms remain unclear, early separation in combination with an angry temperament may increase risk for the cognitive and perceptual disturbances that are more characteristic of schizotypal pathology. Increased risk for BPD may be more specifically linked with affect dysregulation. Future developmental investigations may aid understanding of mechanisms of this risk by examining early separation as a risk for other disorders (e.g., depression, anxiety, and disruptive behavior disorders), alternative dimensional conceptualizations of psychopathology (internalizing and externalizing symptoms), or specific high-risk behaviors (e.g., suicide attempts).

\section{Significance and Limitations}

Perhaps it is most significant that early separations had enduring effects on the developmental trajectory of BPD symptoms that were independent of a range of other risks that could plausibly be outcomes of those early separations. Some of these risks mediated the effects of other risks but none accounted for the association of early separation with failure to "mature out of" BPD symptoms with age. Although it is noteworthy that this early experience had such an enduring effect, we note no single risk is insufficient on its own to cause BPD and particularly look forward to the genetic information now being gathered on this cohort to determine its potential role in these developmental outcomes.

The longitudinal data in this study represent an important strength. Early separations were reported by mothers when the children were still young. Mothers also reported on childhood temperament when children were on average 9 years old and maternal characteristics and behavior when children were on average 13.7 years old. As such, the developmental data here are unlikely to be distorted by memory and reporting biases associated with self-reported data on childhood gathered retrospectively from adults. Repeated assessments of self-reported BPD symptoms also provide a more reliable and probably more valid measure of this personality disorder in adolescence and adulthood. All data were gathered from two independent informants, mother and child, in in-home or (rarely) telephone interviews or (recently) on-line responses. Nevertheless, the data do not include the structured observations that would strengthen the objectivity of assessments. Further, the principal investigator's interest in a broad range of emotional/cognitive problems and risks led to less intense assessment of individual variables than would usually characterize a cross-sectional or laboratory-based study.

Insofar as our attempt to understand the mechanisms of early separation effects draws heavily on attachment theory, it is limited by its lack of any observational or interview measure of attachment in the parent-child relationship. Without such a measure, it remains unclear whether attachment insecurity experienced in childhood mediates long-term association between early separation and subsequent BPD. Although inclusion of self-reported attachment scales provides relevant information, these measures focus on close relationships in adolescence and adulthood, and thus differ from measures focusing on parent-child attach- 
ment (Bartholomew \& Shaver, 1998; Shaver \& Mikulincer, 2004). At present, the causal direction of effects linking self-reported attachment and BPD symptoms is unclear; both may be influenced by common interpersonal experience. New investigations of the long-term relationship between early separation, childhood attachment insecurity, and adult BPD are needed and may be more feasible now that a sufficient number of children assessed with the Strange Situation procedure have reached adulthood (Waters et al., 2000; Weinfeld, Sroufe, \& Egeland, 2000).

\section{References}

Agrawal, H. R., Gunderson, J., Holmes, B. M., \& Lyons-Ruth, K. (2004). Attachment studies with borderline patients: A review. Harvard Review of Psychiatry, 12, 94-104.

American Psychiatric Association. (1980). Diagnostic and statistical manual of mental disorders (3rd ed.). Washington, DC: Author.

American Psychiatric Association. (1994). Diagnostic and statistical manual of mental disorders (4th ed.). Washington, DC: Author.

Anglin, D. M., Cohen, P. R., \& Chen, H. (2008). Duration of early maternal separation and prediction of schizotypal symptoms from early adolescence to midlife. Schizophrenia Research, 103, 143-150.

Bandelow, B., Krause, J., Wedekind, D., Broocks, A., Hajak, G., \& Ruther, E. (2005). Early traumatic life events, parental attitudes, family history, and birth risk factors in patients with borderline personality disorder and healthy controls. Psychiatry Research, 134, 169-179.

Barry, R. A., Kochanska, G., \& Philibert, R. A. (2008). G× $\mathrm{E}$ interaction in the organization of attachment: Mothers' responsiveness as a moderator of children's genotypes. Journal of Child Psychology and Psychiatry, 49, 1313-1320.

Bartholomew, K., Kwong, M. J., \& Hart, S. D. (2001). Attachment. In W. J. Livesley (Ed.), Handbook of personality disorders (pp. 196-230). New York: Guilford Press.

Bartholomew, K., \& Shaver, P. R. (1998). Methods of assessing adult attachment: Do they converge? In J. A. Simpson \& W. S. Rholes (Eds.), Attachment theory and close relationships (pp. 25-45). New York: Guilford Press.

Belsky, J. (2002). Developmental origins of attachment styles. Attachment and Human Development, 4, 166170.

Berenson, K. R., Crawford, T. N., Cohen, P., \& Brook, J. (2005). Implications of identification with parents and parents' acceptance for adolescent and young adult self-esteem. Self and Identity, 4, 289-301.

Bezirganian, S., Cohen, P., \& Brook, J. (1993). The impact of mother-child interaction on the development of borderline personality disorder. American Journal of Psychiatry, 150, 1836-1842.

Bowlby, J. (1969). Attachment and loss: Vol. 1. Attachment. New York: Basic Books.

Bowlby, J. (1973). Attachment and loss: Vol. 2. Separation. New York: Basic Books.

Bowlby, J. (1980). Attachment and loss: Vol. 3. Loss, sadness, and depression. New York: Basic Books.

Bradley, S. J. (1979). The relationship of early maternal separation to borderline personality in children and adolescents: A pilot study. American Journal of Psychiatry, 136, 424-426.

Brennan, K. A., Clark, C. L., \& Shaver, P. R. (1998). Selfreport measurement of adult attachment: An integrative approach. In J. A. Simpson \& W. S. Rhodes (Eds.), Attachment theory and close relationships (pp. 46-76). New York: Guildford Press.

Brennan, K. A., \& Shaver, P. R. (1998). Attachment styles and personality disorders: Their connection to each other and parental divorce, parental death, and perception of parental caregiving. Journal of Personality, 66, 835-878.

Bretherton, I., \& Munholland, K. A. (1999). Internal working models in attachment relationships: A construct revisited. In J. Cassidy \& P. R. Shaver (Eds.), Handbook of attachment: Theory, research, and clinical applications (pp. 89-111). New York: Guilford Press.

Caspi, A., Sugden, K., Moffitt, T. E., Taylor, A., Craig, I. W., Harrington, H. L., et al. (2003). Influence of life stress on depression: Moderation by a polymorphism in the 5-HTT gene. Science, 301, 386-389.

Charmandari, E., Kino, T., Souvatzoglou, E., \& Chrousos, G. P. (2003). Pediatric stress: Hormonal mediators and human development. Hormone Research, 59, 161-179.

Chen, H., Cohen, P., Crawford, T. N., Kasen, S., Johnson, J. G., \& Berenson, K. (2006). Relative impact of young adult personality disorders on subsequent quality of life: Findings of a community-based longitudinal study. Journal of Personality Disorders. 20, 510-523.

Chen, H., Cohen, P., Johnson, J. G., Kasen, S., Sneed, J. R., \& Crawford, T. N. (2004). Adolescent personality disorders and conflict with romantic partners during the transition to adulthood. Journal of Personality Disorders, 18, 507-525.

Chess, S., \& Thomas, A. (1978). Temperamental individuality from childhood to adolescence. Annual Progress in Child Psychiatry and Child Development, 223-244.

Cicchetti D., \& Cohen D. (Eds.). (2006a). Developmental psychopathology: Vol. 1. Theory and method (2nd ed.). New York: Wiley.

Cicchetti D., \& Cohen D. (Eds.). (2006b). Developmental psychopathology: Vol. 3. Risk, disorder, and adaptation (2nd ed.). New York: Wiley.

Cicchetti, D., \& Rogosch, F. A. (1996). Equifinality and multifinality in developmental psychopathology. Development and Psychopathology, 8, 597-600.

Cohen, P. (1996). Childhood risks for young adult symptoms of personality disorder: Method and substance. Multivariate Behavioral Research, 31, 121-148.

Cohen, P. (2008). Child development and personality disorder. Psychiatric Clinics of North America, 31, 477-493.

Cohen, P., Chen, H., Gordon, K., Johnson, J., Brook, J., \& Kasen, S. (2008). Socioeconomic background and the developmental course of schizotypal and borderline personality disorder symptoms. Development and Psychopathology, 20, 633-650.

Cohen, P., \& Cohen, J. (1996). Life values and adolescent mental health. Mahwah, NJ: Erlbaum.

Cohen, P., \& Crawford, T. N. (2005). Developmental issues. In J. M. Oldham, A. E. Skodol, \& D. S. Bender (Eds.), 
Textbook of personality disorders (pp. 171-185). Washington, DC: American Psychiatric Publishing.

Cohen, P., Crawford, T. N., Johnson, J. G., \& Kasen, S. (2005). The Children in the Community Study of developmental course of personality disorder. Journal of Personality Disorders, 19, 466-486.

Crawford, T. N., Cohen, P., Johnson, J. G., Kasen, S., First, M. B., Gordon, K., et al. (2005). Self-reported personality disorder in the Children in the Community sample: Convergent and prospective validity in late adolescence and adulthood. Journal of Personality Disorders, 19, $30-52$.

Crawford, T. N., Cohen, P., Midlarsky, E., \& Brook, J. S. (2001). Internalizing symptoms in adolescents: Gender differences in vulnerability to parental distress and discord. Journal of Research on Adolescence, 11, 85-119.

Crawford, T. N., Shaver, P. R., Cohen, P., Pilkonis, P. A., Gillath, O., \& Kasen, S. (2006). Self-reported attachment, interpersonal aggression, and personality disorder in a prospective community sample of adolescents and adults. Journal of Personality Disorders, 20, 331-353.

Davies, P. T., \& Cummings, E. M. (1994). Marital conflict and child adjustment: An emotional security hypothesis. Psychological Bulletin, 116, 387-411.

Derogatis, L. R., Lipman, R. S., Rickles, K., Uhlenhuth, E. H., \& Covi, L. (1974). The Hopkins Symptom Checklist (HSCL). Behavioral Science, 19, 1-15.

Fincham, F. D., \& Osborne, L. N. (1993). Marital conflict and children: Retrospect and prospect. Clinical Psychology Review, 13, 75-88.

First, M. B., Gibbon, M., Spitzer, R. L., \& Williams, J. B. W. (1997). Structured Clinical Interview for DSM-IVAxis II personality disorders (SCID-II). Arlington, VA: American Psychiatric Publishing.

Fonagy, P., Gergely, G., Jurist, E. L., \& Target, M. (2002). Affect regulation, mentalization, and the development of the self. New York: Other Press.

Fox, N. A., Nichols, K. E., Henderson, H. A., Rubin, K., Schmidt, L., Hamer, D., et al. (2005). Evidence for a gene-environment interaction in predicting behavioral inhibition in middle childhood. Psychological Science, 16, 921-926.

Garner, B., Chanen, A. M., Phillips, L., Velakoulis, D., Wood, S. J., Jackson, H. J., et al. (2007). Pituitary volume in teenagers with first-presentation borderline personality disorder. Psychiatry Research, 156, 257-261.

George, C., Kaplan, N., \& Main, M. (1985). The Berkeley Adult Attachment Interview. Unpublished manuscript, University of California, Berkeley.

Gross, R., Olfson, M., Gameroff, M., Shea, S., Feder, A. Fuentes, M., et al. (2002). Borderline personality disorder in primary care. Archives of Internal Medicine, 162, $53-60$.

Heim, C., \& Nemeroff, C. (2001). The role of childhood trauma in the neurobiology of mood and anxiety disorders: Preclinical and clinical studies. Biological Psychiatry, 49, 1023-1039.

Holmes, J. (2004). Disorganized attachment and borderline personality disorder: A clinical perspective. Attachment and Human Development, 6, 181-190.

Hyler, S. E., Rieder, R., Spitzer, R., \& Williams, J. (1982). The Personality Diagnostic Questionnaire (PDQ). New York: New York State Psychiatric Institute.

Hyler, S. E., Skodol, A. E., Kellman, H. D., Oldham, J. M., \& Rosnick, L. (1990). Validity of the Personality Diagnostic Questionnaire-Revised: Comparison with two structured interviews. American Journal of Psychiatry, 47, 1043-1048.
Jessor, R., Graves, T. D., Hanson, R. C., \& Jessor, S. L. (1968). Society, personality, and deviant behavior: A study of a tri-ethnic community. New York: Holt, Rinehart \& Wilson.

Johnson, J. G., Cohen, P., Kasen, S., Skodol, A., \& Brook, J. (2000). Age-related change in personality disorder trait levels between early adolescence and adulthood: A community-based longitudinal investigation. Acta Psychiatrica Scandinavica, 102, 265-275.

Johnson, J. G., Cohen, P., Kasen, S., Ehrensaft, M. K., \& Crawford, T. N. (2006). Associations of parental personality disorders and Axis I disorders with childrearing behavior. Psychiatry: Interpersonal and Biological Processes, 69, 336-350.

Kaufman, J., Yang, B., Douglas-Palumberi, H., Grasso, D., Lipschitz, D., Houshyar, S., et al. (2006). Brain-derived neurotrophic factor-5-HTTLPR gene interactions and environmental modifiers of depression in children. Biological Psychiatry, 59, 673-680.

Kaufman, J., Yang, B., Douglas-Palumberi, H., Houshyar, S., Lipschitz, D., Krystal, J. H., et al. (2004). Social supports and serotonin transporter gene moderate depression in maltreated children. Proceedings of the National Academy of Sciences of the United States of America, $101,17316-17321$.

Kavoussi, R. J., \& Siever, L. J. (1992). Overlap between borderline and schizotypal personality disorders. Comprehensive Psychiatry, 33, 7-12.

Lenzenweger, M., Loranger, A., Korfine, L., \& Neff, C. (1997). Detecting personality disorders in a nonclinical population: Application of a 2-stage for case identification. Archives of General Psychiatry, 54, 345-351.

Levy, K. N. (2005). The implications of attachment theory and research for understanding borderline personality disorder. Development and Psychopathology, 17, 959-986.

Lieb, K., Rexhausen, J. E., Kahl, K. G., Schweiger, U., Philipsen, A., Hellhammer, D. H., et al. (2004). Increased diurnal salivary cortisol in women with borderline personality disorder. Journal of Psychiatric Research, 38, $559-565$.

Links, P. S., Steiner, M., Offord, D. R., \& Eppel, A. B (1988). Characteristics of borderline personality disorder: A Canadian study. Canadian Journal of Psychiatry, 33, 336-340.

Maughan, B., Pickles, A., \& Quinton, D. (1995). Parental hostility, childhood behavior and adult social functioning. In J. McCord (Ed.), Coercion and punishment in long term perspectives (pp. 34-58). New York: Cambridge University Press.

Maughan, B., \& Rutter, M. (1997). Retrospective reporting of childhood adversity: Issues in assessing long-term recall. Journal of Personality Disorders, 11, 19-33.

McGlashan, T. (1986). The Chestnut Lodge Follow-Up Study, Part III: Long-term outcome of borderline personalities. Archives of General Psychiatry, 42, 20-30.

Mikulincer, M., \& Shaver, P. R. (2007). Attachment in adulthood: Structure, dynamics, and change. New York: Guilford Press.

Modestin, J., Ern, T., \& Oberson, B. (1998). A comparison of self-report and interview diagnoses of DSM-III-R personality disorders. European Journal of Personality, 12, 445-455.

Moss, E., Cyr, C., Bureau, J.-F., Tarabulsy, G. M., \& DuboisComtois, K. (2005). Stability of attachment during the preschool period. Developmental Psychology, 41, 773-783.

Paris, J., Brown, R., \& Nowlis, D. (1987). Long-term follow-up of borderline patients in a general hospital. Comprehensive Psychiatry, 28, 530-535. 
Paris, J., Zweig-Frank, H., \& Guzder, J. (1994). Psychological risk factors for borderline personality disorder in female patients. Comprehensive Psychiatry, 35, 301-305.

Reich, B., \& Zanarini, M. C. (2001). Developmental aspects of borderline personality disorder. Harvard Review of Psychiatry, 9, 294-301.

Robins, L. N., Schoenberg, S. P., Holmes, S. J., Ratcliff, K. S., Benham, A., \& Works, J. (1985). Early home environment and retrospective recall: A test for concordance between siblings with and without psychiatric disorders. American Journal of Orthopsychiatry, 55, 27-41.

Rothbart, M. K., Ahadi, S. A., Hershey, K. L., \& Fisher, P. (2001). Investigations of temperament at three to seven years: The Children's Behavior Questionnaire. Child Development, 72, 1394-1408.

Rutter, M., \& Sroufe, L. A. (2000). Developmental psychopathology: Concepts and challenges. Development and Psychopathology, 12, 265-296.

Salzman, J. P., Salzman, C., \& Wolfson, A. N. (1997). Relationship of childhood abuse and maternal attachment to the development of borderline personality disorder. In M. C. Zanarini (Ed.), Role of sexual abuse in the etiology of borderline personality disorder (pp. 71-91). Washington, DC: American Psychiatric Press.

Sanislow, C. A., Morey, L. C., Grilo, C. M., Gunderson, J. G., Shea, M. T., Skodol, A. E., et al. (2002). Confirmatory factor analysis of DSM-IV borderline, schizotypal, avoidant and obsessive-compulsive personality disorders: Findings from the Collaborative Longitudinal Personality Disorders Study. Acta Psychiatrica Scandinavica, 105, 28-36.

Schaefer, E. (1965). Children's reports of parental behavior: An inventory. Child Development, 36, 413-424.

Shaver, P. R., \& Mikulincer, M. (2002). Attachment-related psychodynamics. Attachment and Human Development, 4, 133-161.

Shaver, P. R., \& Mikulincer, M. (2004). What do self-report attachment measures assess? In W. S. Rholes \& J. A. Simpson (Eds.), Adult attachment: Theory, research, and clinical implications (pp. 17-54). New York: Guilford Press.

Shea, M. T., Stout, R., Gunderson, J., Morey, L. C., Grilo, C. M., McGlashan, T., et al. (2002). Short-term diagnostic stability of schizotypal, borderline, avoidant, and obsessive-compulsive personality disorders. American Journal of Psychiatry, 159, 2036-2041.

Siever, L. J., \& Davis, K. L. (1991). A psychobiological perspective on the personality disorders. American Journal of Psychiatry, 148, 1647-1658.

Smith, S. M., \& Vale, W. W. (2006). The role of the hypothalamic-pituitary-adrenal axis in neuroendocrine responses to stress. Dialogues in Clinical Neuroscience, 8, 383-395.

Skodol, A. E., \& Bender, D. S. (2003). Why are women diagnosed borderline more than men? Psychiatric Quarterly, 74, 349-360.
Skodol, A. E., Gunderson, J. G., Livesley, W. J., Pfohl, B., Siever, L. J., \& Widiger, T. A. (2002). The borderline diagnosis from the perspectives of psychopathology, comorbidity, personality structure, biology, genetics, and course. Biological Psychiatry, 51, 936-950.

Soloff, P. H., \& Millward, J. W. (1983). Developmental histories of borderline patients. Comprehensive Psychiatry, 24, 574-588.

Solomon, J., \& George, C. (1999). Attachment disorganization. New York: Guilford Press.

Stalker, C. A., \& Davies, F. (1995). Attachment organization and adaptation in sexually-abused women. Canadian Journal of Psychiatry, 40, 234-240.

Stone, M. H., Hurt, S. W., \& Stone, D. K. (1987). The P.I. 500: Long-term follow-up of borderline inpatients meeting DSM-III criteria: I. Global outcome. Journal of Personality Disorders, 1, 291-298.

Suomi, S. J. (2006). Risk, resilience, and Gene $\times$ Environment interactions in rhesus monkeys. Annals of the New York Academy of Sciences, 1094, 52-62.

Tarullo, A. R., \& Gunnar, M. R. (2006). Child maltreatment and the developing HPA axis. Hormones and Behavior, 50, 632-639.

Thomas, A., \& Chess, S. (1977). Temperament and development. Oxford: Brunner/Mazel.

Thomas, A., Chess, S., \& Birch, H. G. (1970). The origin of personality. Scientific American, 223, 102-109.

Thomas, A., Chess, S., \& Korn, S. J. (1982). The reality of difficult temperament. Merrill-Palmer Quarterly, 28, 1-20.

Torgersen, S., Kringlen, E., \& Cramer, V. (2001). The prevalence of personality disorders in a community sample. Archives of General Psychiatry, 58, 590-596.

Van Voorhees, E., \& Scarpa, A. (2004). The effects of child maltreatment on the hypothalamic-pituitary-adrenal axis. Trauma Violence and Abuse, 5, 333-352.

Waters, E., Merrick, S., Treboux, D., Crowell, J., \& Albersheim, L. (2000). Attachment security in infancy and early adulthood: A twenty-year longitudinal study. Child Development, 71, 684-689.

Weinfeld, N. S., Sroufe, L. A., \& Egeland, B. (2000). Attachment from infancy to early adulthood in a highrisk sample: Continuity, discontinuity, and their correlates. Child Development, 71, 695-702.

Winograd, G., Cohen, P., \& Chen, H. (2008). Adolescent borderline symptoms in the community: Prognosis for functioning over 20 years. Journal of Child Psychology and Psychiatry, 49, 933-941.

Zanarini, M. C., Yong, L., Frankenburg, F. R., Hennen, J., Reich, D. B., Marino, M. F., \& Vujanovic, A. A. (2002). Severity of reported childhood sexual abuse and its relationship to severity of borderline psychopathology and psychosocial impairment among borderline inpatients. Journal of Nervous and Mental Disease, 190, 381-387. 